\title{
Quantum Speed Limit for Relativistic Spin-0 and Spin-1 Bosons on Commutative and Noncommutative Planes
}

\author{
Kang Wang, ${ }^{1}$ Yu-Fei Zhang, ${ }^{1}$ Qing Wang, ${ }^{2}$ Zheng-Wen Long, ${ }^{3}$ and Jian Jing ${ }^{1}$ \\ ${ }^{1}$ Department of Physics and Electronics, School of Science, Beijing University of Chemical Technology, Beijing 100029, China \\ ${ }^{2}$ College of Physics and Technology, Xinjiang University, Urumqi 830046, China \\ ${ }^{3}$ College of Physics, Guizhou University, Guiyang 550025, China
}

Correspondence should be addressed to Jian Jing; jingjian@mail.buct.edu.cn

Received 15 May 2017; Revised 28 July 2017; Accepted 27 August 2017; Published 22 October 2017

Academic Editor: Shi-Hai Dong

Copyright (C) 2017 Kang Wang et al. This is an open access article distributed under the Creative Commons Attribution License, which permits unrestricted use, distribution, and reproduction in any medium, provided the original work is properly cited. The publication of this article was funded by $\mathrm{SCOAP}^{3}$.

\begin{abstract}
Quantum speed limits of relativistic charged spin-0 and spin-1 bosons in the background of a homogeneous magnetic field are studied on both commutative and noncommutative planes. We show that, on the commutative plane, the average speeds of wave packets along the radial direction during the interval in which a quantum state is evolving from an initial state to the orthogonal final one can not exceed the speed of light, regardless of the intensities of the magnetic field. However, due to the noncommutativity, the average speeds of the wave packets on noncommutative plane will exceed the speed of light in vacuum provided the intensity of the magnetic field is strong enough. It is a clear signature of violating Lorentz invariance in the relativistic quantum mechanics region.
\end{abstract}

\section{Introduction}

Duffin-Kemmer-Petiau (DKP) equation is a first-order relativistic wave equation [1-3]. Different from Dirac equation which describes spin- $1 / 2$ fermions, DKP equation describes spin-0 and spin-1 bosons. DKP equation takes the form

$$
\left(i \beta^{\mu} \partial_{\mu}-m_{0}\right) \psi=0
$$

where $m_{0}$ is the rest mass and the matrices $\beta^{\mu}$ satisfy the algebraic relation

$$
\beta^{\mu} \beta^{\nu} \beta^{\alpha}+\beta^{\alpha} \beta^{\nu} \beta^{\mu}=g^{\mu \nu} \beta^{\alpha}+g^{\alpha \nu} \beta^{\mu} .
$$

Here, $g^{\mu \nu}=g_{\mu \nu}=\operatorname{diag}(+,-,-,-)$ is the metric tensor. The algebra (2) has three different representations: a (onedimensional) trivial representation, a five-dimensional representation describing spin- 0 bosons, and a ten-dimensional representation describing spin-1 bosons. As a Dirac-type relativistic quantum mechanical model, DKP equation with various potentials has been studied in the past years [4-8]. The magnetic coupling in DKP equation is also considered
[9]. When magnetic field is taken into consideration, one should introduce the magnetic potentials by the minimal coupling (we set $\hbar=c=1$ ), $p_{i} \rightarrow p_{i}+q A_{i}$, where $q$ and $A_{i}$ are the charge and the magnetic potentials, respectively. Since DKP equation is analogous with Dirac equation, the author of [9] compares it with Dirac equation in detail in that paper.

On the other hand, the minimum time of a quantum state evolving from an initial state to the orthogonal final one in Hilbert space is of great importance in the field of quantum computation, quantum control, and quantum metrology. In fact, it has attracted attention for a long time [10]. At present, there are two different descriptions of the minimum time for a quantum system evolving from an initial state to the orthogonal final state. One is given by the expression $T_{\min }=\pi \hbar / 2 \Delta E$, in which $\Delta E$ is the energy variance, defined by $\Delta E=\sqrt{\left\langle\psi\left|H^{2}\right| \psi\right\rangle-\langle\psi|H| \psi\rangle^{2}}$, with $H$ being the Hamiltonian of the system and $|\psi\rangle$ being a specific superposition of eigenstates of $H$ [11]. The other is given in [12], which states that the minimum time is given by $T_{\min }=\pi \hbar / 2\left(\bar{E}-E_{0}\right)$, where $\bar{E}$ and $E_{0}$ are the mean 
energy and the lowest energy of the state which participates in the superposition. Obviously, the results of $[11,12]$ will be equivalent if the condition $\Delta E=\bar{E}-E_{0}$ is satisfied. This condition can be simply satisfied by superposing two steady states homogeneously. According to the results in $[11,12]$, it is natural to assume that the minimum time should be given by $T_{\min }=\max \left\{\pi \hbar / 2 \Delta E, \pi \hbar / 2\left(\bar{E}-E_{0}\right)\right\}$ [13]. A unified bound which contains both $\Delta E$ and $\bar{E}$ is considered [14].

An interesting connection between the minimum time of the quantum state evolving in Hilbert space and the average speed of an electron wave packet travelling in spatial space is constructed in a recent paper [15]. In this paper, the authors study a relativistic electron coupling to a homogeneous magnetic field. They find that the average speed of this electron wave packet moving in radial direction during the interval in which a quantum state is evolving from an initial state to the orthogonal final one in Hilbert space is less than the speed of light in vacuum, regardless of intensities of the magnetic field one applies. It seems that, as expected, Lorentz invariance is not violated in this relativistic quantum mechanical model. However, Lorentz invariance would be violated in the nonrelativistic limit of this model since the average speed of the electron wave packet in radial direction exceeds the speed of light in vacuum provided the intensity of the magnetic field is strong enough.

The work of [15] is generalized to noncommutative case recently. Noncommutativity becomes one of the foci of theoretical research due to superstring theories in recent years [16-19]. There are a large number of papers studying quantum field theories in noncommutative space [2025]. Nonrelativistic noncommutative quantum mechanical models, such as noncommutative harmonic oscillator and noncommutative Landau problem, have been studied extensively [26-32]. The relativistic quantum mechanical models on noncommutative space are investigated since the work of [33]. Some geometrical phases in noncommutative relativistic quantum theory are studied in [34-36] recently. DKP equation in noncommutative space, especially the DKP oscillator in noncommutative space, has also been investigated by some authors [37-42]. Interestingly, it is found that the noncommutativity even has some relationships with Jaynes-Cumming model in quantum optics context [43].

In [44], the authors study the noncommutative (both the coordinates and momenta are noncommutative simultaneously) Dirac equation. They find that Lorentz invariance will be violated in noncommutative Dirac equation since the average speed of an electron wave packet exceeds the speed of light in vacuum if the magnetic field is strong enough. In fact, the problem of violating Lorentz invariance has been considered [45-47]. In [46], the authors find that because of noncommutativity, Lorentz invariance will be violated in the noncommutative quantum electrodynamics (QED) since the electromagnetic wave travels in different speeds along different directions at the presence of a background magnetic field. A similar result is also obtained in [47]. This problem is also considered semiclassically from both noncommutative and gravitational points of view [48-50].
In this paper, we investigate the problems of whether Lorentz invariance is violated for spin- 0 or spin-1 relativistic bosons in two-dimensional spatial space. We study the commutative case firstly and then generalize to the noncommutative case. The organization of this paper is as follows. In the next section, we study spin- 0 and spin- 1 charged bosons coupling to homogeneous magnetic fields on commutative plane. Then, in Section 3, we study the noncommutative case. Some remarks and further discussions will be presented in the last section.

\section{Spin-0 and Spin-1 Charged Bosons Coupling to a Homogenous Magnetic Field on Commutative Plane}

In this section, we study spin- 0 and spin-1 bosons coupling to a homogeneous magnetic field on a commutative plane. We start our studies from the spin-0 bosons.

As stated before, the five-dimensional representation of the algebra (2) describes spin-0 boson. The explicit expressions of five-dimensional $\beta^{\mu}$ matrices are

$$
\begin{aligned}
& \beta^{0}=\left(\begin{array}{cc}
\Theta & \overline{0} \\
\overline{0}^{T} & 0
\end{array}\right), \\
& \beta^{i}=\left(\begin{array}{cc}
\tilde{0} & \rho^{i} \\
\left(-\rho^{i}\right)^{T} & 0
\end{array}\right),
\end{aligned}
$$

in which

$$
\begin{aligned}
\Theta & =\left(\begin{array}{ll}
0 & 1 \\
1 & 0
\end{array}\right), \\
\rho^{1} & =\left(\begin{array}{ccc}
-1 & 0 & 0 \\
0 & 0 & 0
\end{array}\right), \\
\rho^{2} & =\left(\begin{array}{ccc}
0 & -1 & 0 \\
0 & 0 & 0
\end{array}\right), \\
\rho^{3} & =\left(\begin{array}{ccc}
0 & 0 & -1 \\
0 & 0 & 0
\end{array}\right) .
\end{aligned}
$$

Here $\overline{0}, \widetilde{0}$, and 0 are $2 \times 3,2 \times 2$ and $3 \times 3$ zero matrices, respectively. Choosing symmetric gauge $A_{i}=-(1 / 2) B \epsilon_{i j} x_{j}$ and introducing Larmor frequency $\omega_{L}=q B / 2 m_{0}$, we write (1) in the form

$$
\begin{aligned}
& {\left[\beta^{0} E+\beta^{1}\left(p_{1}-m_{0} \omega_{L} x_{2}\right)+\beta^{2}\left(p_{2}+m_{0} \omega_{L} x_{1}\right)-m_{0}\right]} \\
& \cdot \psi\left(x_{1}, x_{2}, t\right)=0,
\end{aligned}
$$

where $\psi\left(x_{1}, x_{2}, t\right)$ is a five-component wave function $\psi\left(x_{1}, x_{2}, t\right)=\left(\psi^{1}, \psi^{2}, \psi^{3}, \psi^{4}, \psi^{5}\right)^{T}\left(x_{1}, x_{2}, t\right)$. 
Substituting the explicit expressions of $\beta^{\mu}$ matrices (3) into the above equation, we get a set of equations

$$
\begin{aligned}
& -m_{0} \psi^{1}+E \psi^{2}-\left(p_{1}-m_{0} \omega_{L} x_{2}\right) \psi^{3} \\
& \quad-\left(p_{2}+m_{0} \omega_{L} x_{1}\right) \psi^{4}=0 \\
& E \psi^{1}-m_{0} \psi^{2}=0 \\
& \left(p_{1}-m_{0} \omega_{L} x_{2}\right) \psi_{1}-m_{0} \psi^{3}=0 \\
& \left(p_{2}+m_{0} \omega_{L} x_{1}\right) \psi_{1}-m_{0} \psi^{4}=0 \\
& \psi^{5}=0
\end{aligned}
$$

Obviously, the five components $\left(\psi^{1}, \psi^{2}, \psi^{3}, \psi^{4}, \psi^{5}\right)$ are not independent to each other.

Combining the above equations, we get the dynamical equation of the component $\psi^{1}$. It is

$$
\left(E^{2}-m_{0}^{2}\right) \psi^{1}=2 m_{0} H_{L} \psi^{1},
$$

where $H_{L}$ is the Landau Hamiltonian:

$$
H_{L}=\frac{1}{2 m_{0}}\left(p_{1}^{2}+p_{2}^{2}\right)+\frac{1}{2} m_{0} \omega_{L}^{2}\left(x_{1}^{2}+x_{2}^{2}\right)+\omega_{L} L_{z},
$$

with $L_{z}=x p_{y}-y p_{x}$ being the angular momentum along $z$ direction. Obviously, the Hamiltonian (8) describes a planar nonrelativistic charged particle interacting with a homogeneous perpendicular magnetic field.

Equation (7) is easily solved. The eigenvalues and eigenfunctions, respectively, are [51]

$$
\begin{aligned}
E_{n, m_{l}}= & \pm \sqrt{m_{0}^{2}+2 m_{0}\left(n+m_{l}+1\right) \omega_{L}}, \\
\psi^{1}= & F_{n, m_{l}}(r, \varphi) \\
= & \frac{(-1)^{\left(n-\left|m_{l}\right|\right) / 2}\left(\left(n-\left|m_{l}\right|\right) / 2\right) !}{\sqrt{\pi\left(\left(n+\left|m_{l}\right|\right) / 2\right) !\left(\left(n-\left|m_{l}\right|\right) / 2\right) !}} \\
& \times \alpha(\alpha r)^{\left|m_{l}\right|} L_{\left(\left(n-\left|m_{l}\right|\right) / 2\right)}^{\left|m_{l}\right|}\left(\alpha^{2} r^{2}\right) e^{-(1 / 2) \alpha^{2} r^{2}} e^{i m_{l} \varphi},
\end{aligned}
$$

where $n=0,1,2, \ldots, m_{l}=-n,-n+2, \ldots, n-2, n, \alpha=$ $\sqrt{m_{0} \omega_{L}}=\sqrt{q B / 2}$, and $L_{\left(\left(n-\mid m_{l}\right) \mid / 2\right)}^{\left|m_{l}\right|}$ is Laguerre's polynomials. Thus, the multicomponent wave function $\psi$ can be obtained if one chooses a specific solution of the component $\psi^{1}$.

In order to simplify our calculation further and make a comparison with the spin-1/2 case [15], we choose two steady states whose first components, respectively, are (we only consider the positive-energy sector)

$$
\begin{aligned}
& \psi^{1}=F_{0,0}(r, \varphi)=\frac{1}{\sqrt{\pi}} \alpha e^{-(1 / 2) \alpha^{2} r^{2}}, \\
& \psi^{1}=F_{2,0}=-\frac{1}{\sqrt{\pi}} \alpha\left(1-\alpha^{2} r^{2}\right) e^{-(1 / 2) \alpha^{2} r^{2}} .
\end{aligned}
$$

Then, after some direct calculations, we get these two steady states. They are

$$
\phi_{0,0}=N_{0,0}\left(\begin{array}{c}
F_{0,0} \\
\frac{E_{0,0}}{m_{0}} F_{0,0} \\
\frac{i \alpha}{m_{0}} F_{1,1} \\
\frac{\alpha}{m_{0}} F_{1,1} \\
0
\end{array}\right) e^{-i E_{0,0} t},
$$

where

$$
\begin{aligned}
& N_{0,0}=\frac{m_{0}}{\sqrt{m_{0}^{2}+E_{0,0}^{2}+2 \alpha^{2}}}, \\
& N_{2,0}=\frac{m_{0}}{\sqrt{m_{0}^{2}+E_{2,0}^{2}+6 \alpha^{2}}}
\end{aligned}
$$

are two normalization constants.

As we have mentioned, there are some controversies on the minimum time of a quantum state evolving from an initial state to the orthogonal one. In order to avoid these problems, we superpose these two steady states (13) homogeneously; that is,

$$
\begin{aligned}
& \Psi(r, \varphi, t) \\
& \quad=\frac{1}{\sqrt{2}}\left[\phi_{0,0}(r, \varphi) e^{-i E_{0,0} t}+\phi_{2,0}(r, \varphi) e^{-i E_{2,0} t}\right] .
\end{aligned}
$$

According to $[11,12]$, the minimum time for a state $\Psi(r, \varphi, t)$ evolving from the initial state $\Psi(r, \varphi, 0)$ to the final orthogonal state $\Psi\left(r, \varphi, T_{\min }\right)$ is given by $T_{\min }=\pi \hbar / 2(\bar{E}-$ $\left.E_{0,0}\right)$, or, equivalently, $T_{\min }=\pi \hbar / 2 \Delta E$, where $\bar{E}$ and $\Delta E$ are the mean energy and energy variance on the state $|\Psi(t)\rangle$, respectively. After direct calculation, we get

$$
T_{\min }=\frac{\pi}{\sqrt{m_{0}^{2}+3 q B}-\sqrt{m_{0}^{2}+q B}} .
$$

The average radial displacement of the spinless boson in the interval $\left[0, T_{\min }\right]$ is given by

$$
\begin{aligned}
\Delta r & =\left|\left\langle\Psi\left(T_{\min }\right)|r| \Psi\left(T_{\min }\right)\right\rangle-\langle\Psi(0)|r| \Psi(0)\rangle\right| \\
& =2\left|\left\langle\phi_{0,0}|r| \phi_{2,0}\right\rangle\right| .
\end{aligned}
$$


Substituting (13) and (15) into the above equation and after some direct calculations, we arrive at

$$
\Delta r=\left(\frac{1}{4}+\frac{3 \sqrt{3}}{8}\right) \frac{\sqrt{\pi}}{\alpha} .
$$

Thus, the average speed of the wave packet of this charged boson along the radial direction during this interval is given by $\bar{v}=\Delta r / T_{\min }$. The result is

$$
\bar{v}=\frac{2+3 \sqrt{3}}{8} \sqrt{\frac{2}{\pi q B}}\left(\sqrt{m_{0}^{2}+3 q B}-\sqrt{m_{0}^{2}+q B}\right) .
$$

The average speed $\bar{v}$ reaches its maximum when $B \rightarrow \infty$. It is

$$
\bar{v}_{\text {max }}=\lim _{B \rightarrow \infty} \bar{v} \doteq 0.5254<1 .
$$

It shows that the average radial speed of this spinless boson wave packet is less than the speed of light in vacuum ( $c=$ 1) no matter the intensities of the magnetic field. Therefore, Lorentz invariance is not violated in this relativistic quantum mechanics model.

Now, we study the DKP equation with 10-dimensional representation of the algebra (2). As is known, it describes spin-1 bosons. The explicit expressions of $\beta$ matrices are

$$
\begin{aligned}
& \beta^{0}=\left(\begin{array}{cccc}
0 & \check{0} & \check{0} & \check{0} \\
\check{0}^{T} & 0_{3 \times 3} & I_{3 \times 3} & 0_{3 \times 3} \\
\check{0}^{T} & I_{3 \times 3} & 0_{3 \times 3} & 0_{3 \times 3} \\
\check{0}^{T} & 0_{3 \times 3} & 0_{3 \times 3} & 0_{3 \times 3}
\end{array}\right), \\
& \beta^{i}=\left(\begin{array}{cccc}
0 & \check{0} & e_{i} & \check{0} \\
\check{0}^{T} & 0_{3 \times 3} & 0_{3 \times 3} & -i S_{i} \\
-e_{i}^{T} & 0_{3 \times 3} & 0_{3 \times 3} & 0_{3 \times 3} \\
\check{0}^{T} & -i S_{i} & 0_{3 \times 3} & 0_{3 \times 3}
\end{array}\right),
\end{aligned}
$$

in which

$$
\begin{aligned}
& \check{0}=(0,0,0), \\
& e_{1}=(1,0,0), \\
& e_{2}=(0,1,0), \\
& e_{3}=(0,0,1), \\
& S_{1}=\left(\begin{array}{lll}
0 & 0 & 0 \\
0 & 0 & -i \\
0 & i & 0
\end{array}\right), \\
& S_{2}=\left(\begin{array}{ccc}
0 & 0 & i \\
0 & 0 & 0 \\
-i & 0 & 0
\end{array}\right), \\
& S_{3}=\left(\begin{array}{lll}
0 & -i & 0 \\
i & 0 & 0 \\
0 & 0 & 0
\end{array}\right) .
\end{aligned}
$$

Using the explicit expressions of $\beta$ matrices, we write the DKP equation in the component form

$$
\begin{aligned}
& \left(p_{1}-m_{0} \omega_{L} x_{2}\right) \psi^{5}+\left(p_{2}+m_{0} \omega_{L} x_{1}\right) \psi^{6}=m_{0} \psi^{1}, \\
& E \psi^{5}+\left(p_{2}+m_{0} \omega_{L} x_{1}\right) \psi^{10}=m_{0} \psi^{2}, \\
& E \psi^{6}-\left(p_{1}-m_{0} \omega_{L} x_{2}\right) \psi^{10}=m_{0} \psi^{3}, \\
& E \psi^{7}-\left(p_{2}+m_{0} \omega_{L} x_{1}\right) \psi^{8}+\left(p_{1}-m_{0} \omega_{L} x_{2}\right) \psi^{9} \\
& \quad=m_{0} \psi^{4}, \\
& E \psi^{2}-\left(p_{1}-m_{0} \omega_{L} x_{2}\right) \psi^{1}=m_{0} \psi^{5}, \\
& E \psi^{3}-\left(p_{2}+m_{0} \omega_{L} x_{1}\right) \psi^{1}=m_{0} \psi^{6}, \\
& E \psi^{4}=m_{0} \psi^{7}, \\
& \left(p_{2}+m_{0} \omega_{L} x_{1}\right) \psi^{4}=m_{0} \psi^{8}, \\
& -\left(p_{1}-m_{0} \omega_{L} x_{1}\right) \psi^{4}=m_{0} \psi^{9}, \\
& -\left(p_{2}+m_{0} \omega_{L} x_{1}\right) \psi^{2}+\left(p_{1}-m_{0} \omega_{L} x_{2}\right) \psi^{3}=m_{0} \psi^{10} .
\end{aligned}
$$

According to (23d), (23g), (23h), and (23i), we get the dynamical equation of the component $\psi^{4}$

$$
\left(E^{2}-m_{0}^{2}\right) \psi^{4}=2 m_{0} H_{L} \psi^{4},
$$

where $H_{L}$ is given in (8).

Therefore, the solutions of $\psi^{4}$ in spin-1 case take the same form as (9) and (10); that is,

$$
\begin{aligned}
E_{n, m_{l}}= & \pm \sqrt{m_{0}^{2}+2 m_{0}\left(n+m_{l}+1\right) \omega_{L}}, \\
\psi^{4}= & F_{n, m_{l}}(r, \varphi) \\
= & \frac{(-1)^{\left(n-\left|m_{l}\right|\right) / 2}\left(\left(n-\left|m_{l}\right|\right) / 2\right) !}{\sqrt{\pi\left(\left(n+\left|m_{l}\right|\right) / 2\right) !\left(\left(n-\left|m_{l}\right|\right) / 2\right) !}} \\
& \times \alpha(\alpha r)^{\left|m_{l}\right|} L_{\left(\left(n-\left|m_{l}\right|\right) / 2\right)}^{\left|m_{l}\right|}\left(\alpha^{2} r^{2}\right) e^{-(1 / 2) \alpha^{2} r^{2}} e^{i m_{l} \varphi} .
\end{aligned}
$$

We choose a special solution for components $\psi^{2}$ and $\psi^{3}$, that is, $\psi^{2}=0$ and $\psi^{3}=0$. As a result, the components $\psi^{1}=$ $\psi^{5}=\psi^{6}=0$. Thus, the ten-component eigenfunction can be obtained if we select a specific solution for $\psi^{4}$ from (26). In order to simplify our calculations and make a comparison with [15], we choose two specific solutions for $\psi^{4}$. They are

$$
\begin{aligned}
& \psi^{4}=F_{0,0}=\frac{1}{\sqrt{\pi}} \alpha e^{-(1 / 2) \alpha^{2} r^{2}}, \\
& \psi^{4}=F_{2,0}=-\frac{1}{\sqrt{\pi}} \alpha\left(1-\alpha^{2} r^{2}\right) e^{-(1 / 2) \alpha^{2} r^{2}} .
\end{aligned}
$$


Thus, the explicit expressions for two steady states we want to superpose are

$$
\begin{aligned}
& \phi_{0,0}=N_{0,0}\left(\begin{array}{c}
0 \\
0 \\
0 \\
F_{0,0} \\
0 \\
0 \\
\frac{E_{0,0}}{m_{0}} F_{0,0} \\
\frac{\alpha}{m_{0}} F_{1,1} \\
-\frac{i \alpha}{m_{0}} F_{1,1} \\
0
\end{array}\right) \text {, } \\
& \phi_{2,0}=N_{2,0}\left(\begin{array}{c}
0 \\
0 \\
0 \\
F_{2,0} \\
0 \\
0 \\
\frac{E_{2,0}}{m_{0}} F_{2,0} \\
\frac{\alpha}{m_{0}}\left(\sqrt{2} F_{3,1}+F_{1,-1}\right) \\
-\frac{i \alpha}{m_{0}}\left(\sqrt{2} F_{3,1}-F_{1,-1}\right) \\
0
\end{array}\right) \text {, }
\end{aligned}
$$

where $N_{0,0}, N_{2,0}$ are identical to (14).

We superpose these two steady states (28) homogeneously in the same manner as (15). The eigenvalues are equal to the ones in spin- 0 case; thus the minimum time for the superposition state evolving from the initial state $\Psi(r, \varphi, 0)$ to the final orthogonal state $\Psi\left(r, \varphi, T_{\min }\right)$ is the same as (16). The radial displacement of the wave packet during the interval $\left[0, T_{\min }\right]$ can be calculated straightforwardly. The result is identical to (17). Therefore, the average velocity along radial direction of this spin-1 boson is the same as in (19). The maximum average speed is achieved when the intensity of the magnetic field tends to infinity. The result is identical to (20). It is less than the speed of light in vacuum. Thus, it shows that Lorentz invariance is not violated.

\section{Spin-0 and Spin-1 Bosons on Noncommutative Plane}

As we have shown, the average speeds of the wave packets of spin- 0 and spin- 1 bosons along the radial direction will not exceed the speed of light in vacuum, regardless of the intensity of the magnetic field. A natural question is does the Lorentz invariance get violated in the noncommutative DKP equation? In the following, we will investigate DKP equation in noncommutative $2+1$-dimensional phase space.

There are two ways to study noncommutative theories in noncommutative space. One is to replace the ordinary product by the Moyal (*) product [52]:

$$
(f * g)\left(x_{I}\right)=f\left(x_{I}\right) e^{(i / 2)\left(\overleftarrow{\partial} / \partial x_{I}\right) \theta^{I J}\left(\vec{\partial} / \partial x_{J}\right)} g\left(x_{I}\right)
$$

where $f, g$ are two $N$-dimensional functions and $\theta^{I J}, I, J=$ $1,2, \ldots, N$ is a rank-two antisymmetrical tensor. When the noncommutativity between momenta is taken into account, the above $*$-product should be generalized to the form

$$
\begin{aligned}
& (f * g)\left(x_{I}, p_{I}\right)=f\left(x_{I}, p_{J}\right) \\
& \cdot \exp \left[\frac{i}{2}\left(\frac{\overleftarrow{\partial}}{\partial x_{I}} \theta^{I J} \frac{\vec{\partial}}{\partial x_{J}}+\frac{\overleftarrow{\partial}}{\partial p_{I}} \eta^{I J} \frac{\vec{\partial}}{\partial p_{J}}\right)\right] g\left(x_{I}, p_{I}\right)
\end{aligned}
$$

in which $\eta^{I J}, I, J=1,2, \ldots, N$ is also a rank-two antisymmetrical tensor.

The other equivalent way is to introduce the noncommutative algebra among variables $\left(x_{i}, p_{i}\right)$. For the noncommutative $2+1$-dimensional phase space, the noncommutative algebra is given by

$$
\begin{aligned}
& {\left[\widehat{x}_{i}, \widehat{x}_{j}\right]=i \theta \epsilon_{i j},} \\
& {\left[\widehat{p}_{i}, \widehat{p}_{j}\right]=i \eta \epsilon_{i j},}
\end{aligned}
$$

$$
i, j=1,2 \text {, }
$$

where $\theta$ and $\eta$ are two real parameters and $\epsilon_{i j}$ is the 2dimensional antisymmetric tensor. It can be easily verified that the $*$-product $(30)$ can reproduce the commutator (31) directly for 2-dimensional phase space.

For the sake of consistency, the commutator among coordinates and momenta should be modified as [53] (in order to avoid the problem of unitarity, we only consider noncommutativity among coordinates and momenta)

$$
\left[\widehat{x}_{i}, \widehat{p}_{j}\right]=i\left(1+\frac{\theta \eta}{4}\right) \delta_{i j}
$$

By applying the latter way to study the noncommutative theories, one assumes that the dynamical equations in noncommutative quantum mechanics take the same form as their commutative counterparts. However, variables in dynamical equation are replaced by the corresponding noncommutative ones. Therefore, the noncommutative version of spin-0 DKP equations is nothing but to replace the variables $\left(x_{i}, p_{i}\right)$ by $\left(\widehat{x}_{i}, \widehat{p}_{i}\right)$ which satisfy the algebraic relations (31) and (32) in (6a), (6b), (6c), (6d), and (6e). Choosing the symmetric gauge $\widehat{A}_{i}=-(B / 2) \epsilon_{i j} \widehat{x}_{j}[54]$ and combining them, we get

$$
\left(E^{2}-m_{0}^{2}\right) \psi^{1}=2 m_{0} \widehat{H}_{L}^{N C} \psi^{1}
$$


where

$$
\widehat{H}_{L}^{N C}=\frac{1}{2 m_{0}}\left(\widehat{p}_{1}^{2}+\widehat{p}_{2}^{2}\right)+\frac{1}{2} m_{0} \omega_{L}^{2}\left(\widehat{x}_{1}^{2}+\widehat{x}_{2}^{2}\right)+\omega_{L} \widehat{L}_{z}
$$

with $\widehat{L}_{z}=\widehat{x} \widehat{p}_{y}-\widehat{y} \widehat{p}_{x}$ being the noncommutative angular momentum along $z$ direction. Hamiltonian (34) describes a planar nonrelativistic charged particle interacting with a homogeneous perpendicular magnetic field on the noncommutative planes (31) and (32).

We map noncommutative variables $\left(\widehat{x}_{i}, \widehat{p}_{\mathrm{i}}\right)$ to commutative ones $\left(x_{i}, p_{i}\right)$ which satisfy the standard Heisenberg algebra

$$
\begin{aligned}
& {\left[x_{i}, x_{j}\right]=\left[p_{i}, p_{j}\right]=0,} \\
& {\left[x_{i}, p_{j}\right]=i \delta_{i j} .}
\end{aligned}
$$

It is straightforward to check that the map from noncommutative variables to commutative ones can be realized by [53]

$$
\begin{aligned}
& \widehat{x}_{i}=x_{i}-\frac{\theta}{2} \epsilon_{i j} p_{j} \\
& \widehat{p}_{i}=p_{i}+\frac{\eta}{2} \epsilon_{i j} x_{j} .
\end{aligned}
$$

In terms of commutative variables $\left(x_{i}, p_{i}\right)$, we find that the dynamical equation for component $\psi^{1}$ takes the same form as (33). The corresponding Hamiltonian (34) in terms of commutative variables becomes

$$
\begin{aligned}
H_{L}^{N C}= & \frac{1}{2 M_{\mathrm{eff}}}\left(p_{1}^{2}+p_{2}^{2}\right)+\frac{1}{2} M_{\mathrm{eff}} \Omega^{2}\left(x_{1}^{2}+x_{2}^{2}\right) \\
& +\Omega L_{z},
\end{aligned}
$$

in which

$$
\begin{aligned}
M_{\mathrm{eff}} & =\frac{m_{0}}{\kappa^{2}}, \\
\Omega^{2} & =\left(\frac{1}{m_{0}} \kappa \gamma\right)^{2},
\end{aligned}
$$

where $\kappa=1-q \theta B / 4, \gamma=q B / 2-\eta / 2$ are two parameters. are

The eigenvalues and eigenfunctions of Hamiltonian (37)

$$
\begin{aligned}
& E_{n, m_{l}}= \pm \sqrt{m_{0}^{2}+2 m_{0}\left(n+m_{l}+1\right) \Omega}, \\
& \psi^{1}=F_{n, m_{l}}(r, \varphi) \\
& =\frac{(-1)^{\left(n-\left|m_{l}\right|\right) / 2}\left(\left(n-\left|m_{l}\right|\right) / 2\right) !}{\sqrt{\pi\left(\left(n+\left|m_{l}\right|\right) / 2\right) !\left(\left(n-\left|m_{l}\right|\right) / 2\right) !}} \\
& \quad \times \alpha^{\prime}\left(\alpha^{\prime} r\right)^{\left|m_{l}\right|} L_{\left(\left(n-\left|m_{l}\right|\right) / 2\right)}^{\left|m_{l}\right|}\left(\alpha^{\prime 2} r^{2}\right) e^{-(1 / 2) \alpha^{\prime 2} r^{2}} e^{i m_{l} \varphi},
\end{aligned}
$$

where $n=0,1,2, \ldots, m_{l}=-n,-n+2, \ldots, n-2, n$ and

$$
\alpha^{\prime}=\sqrt{M_{\mathrm{eff}} \Omega}
$$

Other components can be calculated directly from the commutative version of (6a), (6b), (6c), (6d), and (6e).

In order to make a comparison with commutative version, we choose two solutions of $\psi^{1}$ as

$$
\begin{aligned}
& \psi^{1}=F_{0,0}=\frac{1}{\sqrt{\pi}} \alpha^{\prime} e^{-(1 / 2) \alpha^{\prime 2} r^{2}}, \\
& \psi^{1}=F_{2,0}=-\frac{1}{\sqrt{\pi}} \alpha^{\prime}\left(1-\alpha^{\prime 2} r^{2}\right) e^{-(1 / 2) \alpha^{\prime 2} r^{2}} .
\end{aligned}
$$

Then, the corresponding two steady states are

$$
\phi_{0,0}=N_{0,0}\left(\begin{array}{c}
F_{0,0} \\
\frac{E_{0,0}}{m_{0}} F_{0,0} \\
i \frac{\alpha^{\prime} \kappa}{m_{0}} F_{1,1} \\
\frac{\alpha^{\prime} \kappa}{m_{0}} F_{1,1} \\
0
\end{array}\right),
$$

where $N_{0,0}$ and $N_{2,0}$ are two normalization constants. They are

$$
\begin{aligned}
& N_{0,0}=\frac{m_{0}}{\sqrt{m_{0}^{2}+E_{0,0}^{2}+2 \alpha^{\prime 2} \kappa^{2}}}, \\
& N_{2,0}=\frac{m_{0}}{\sqrt{m_{0}^{2}+E_{2,0}^{2}+6 \alpha^{\prime 2} \kappa^{2}}},
\end{aligned}
$$

respectively.

We superpose two steady states (43) homogeneously in the same manner as (15). According to [11, 12], the minimum time for the superposition state evolving from the initial state $\Psi(r, \varphi, 0)$ to the final orthogonal one $\Phi\left(r, \varphi, T_{\min }\right)$ is given by $T_{\min }=\pi \hbar / 2\left(\bar{E}-E_{0,0}\right)=\pi \hbar / 2 \Delta E$. Substituting the eigenvalues (39) for states $\phi_{0,0}$ and $\phi_{2,0}$ into the expression of the minimum time, we get

$$
T_{\min }=\frac{\pi}{\sqrt{m_{0}^{2}+6 m_{0} \Omega}-\sqrt{m_{0}^{2}+2 m_{0} \Omega}} .
$$

Accordingly, the displacement along the radial direction during the period of time $T_{\min }$ is given by the expression $\Delta r=\left|\left\langle\Psi\left(T_{\min }\right)|r| \Psi\left(T_{\min }\right)\right\rangle-\langle\Psi(0)|r| \Psi(0)\rangle\right|=2\left|\left\langle\phi_{0,0}|r| \phi_{2,0}\right\rangle\right|$. 
Substituting this superposition state into the expressions of $\Delta r$ and taking the limit of $B \rightarrow \infty$, we get

$$
\Delta r=\left|\left\langle\phi_{0,0}|r| \phi_{2,0}\right\rangle\right|=\frac{2+3 \sqrt{3}}{8} \alpha^{\prime} .
$$

Therefore, the average speed along the radial direction of wave packet during the interval $\left[0, T_{\min }\right]$ in the limit $B \rightarrow \infty$ is given by

$$
\bar{v}=\frac{\Delta r}{T_{\min }} \doteq 0.5254|\kappa|
$$

Compared with the commutative case, we find that due to spatial noncommutativity, there is an extra factor $|\kappa|=$ $|1-q \theta B / 4|$. It is this factor which enables average radial speed of the wave packet to exceed the speed of light in vacuum provided the intensity of the magnetic field is strong enough. It is a clear evidence of violating Lorentz invariance in this noncommutative relativistic quantum mechanical model.

Now, we study the noncommutative spin-1 DKP equation. In noncommutative plane, the dynamical equations take the same form as (23a), (23b), (23c), (23d), (23e), (23f), (23g), (23h), (23i), and (23j) except that variables $\left(x_{i}, p_{i}\right)$ are replaced by noncommutative ones $\left(\widehat{x}_{i}, \widehat{p}_{i}\right)$.

Mapping noncommutative variables $\left(\hat{x}_{i}, \widehat{p}_{i}\right)$ to commutative ones $\left(x_{i}, p_{i}\right)$, we get

$$
\begin{aligned}
\left(\kappa p_{1}-\gamma x_{2}\right) \psi^{5}+\left(\kappa p_{2}+\gamma x_{1}\right) \psi^{6} & =m_{0} \psi^{1}, \\
E \psi^{5}+\left(\kappa p_{2}+\gamma x_{1}\right) \psi^{10} & =m_{0} \psi^{2}, \\
E \psi^{6}-\left(\kappa p_{1}-\gamma x_{2}\right) \psi^{10} & =m_{0} \psi^{3} \\
E \psi^{7}-\left(\kappa p_{2}+\gamma x_{1}\right) \psi^{8}+\left(\kappa p_{1}-\gamma x_{2}\right) \psi^{9} & =m_{0} \psi^{4}, \\
E \psi^{2}-\left(\kappa p_{1}-\gamma x_{2}\right) \psi^{1} & =m_{0} \psi^{5} \\
E \psi^{3}-\left(\kappa p_{2}+\gamma x_{1}\right) \psi^{1} & =m_{0} \psi^{6} \\
E \psi^{4} & =m_{0} \psi^{7} \\
\left(\kappa p_{2}+\gamma x_{1}\right) \psi^{4} & =m_{0} \psi^{8} \\
-\left(\kappa p_{1}-\gamma x_{2}\right) \psi^{4} & =m_{0} \psi^{9} \\
-\left(\kappa p_{2}+\gamma x_{1}\right) \psi^{2}+\left(\kappa p_{1}-\gamma x_{2}\right) \psi^{3} & =m_{0} \psi^{10}
\end{aligned}
$$

The dynamical equation for component $\psi^{4}$ can be obtained from above equations. It is

$$
\left(E^{2}-m_{0}^{2}\right) \psi^{4}=2 m_{0} H_{L}^{N C} \psi^{4}
$$

where $H_{L}^{N C}$ is given in (37). The solutions to (49) can be obtained in the standard way. The eigenvalues are identical to (39) and the corresponding solutions of component $\psi^{4}$ are identical with the solutions of $\psi^{1}$ in noncommutative spin- 0 case (40).

Similar with the commutative version, we set $\psi^{2}=\psi^{3}=$ 0 . Then the other components can be determined by (48a), (48b), (48c), (48d), (48e), (48f), (48g), (48h), (48i), and (48j). For the sake of comparing with the corresponding commutative case, we choose two specific solutions of $\psi^{4}$ :

$$
\begin{aligned}
& \psi^{4}=F_{0,0}=\frac{1}{\sqrt{\pi}} \alpha^{\prime} e^{-(1 / 2) \alpha^{\prime 2} r^{2}}, \\
& \psi^{4}=F_{2,0}=-\frac{1}{\sqrt{\pi}} \alpha^{\prime}\left(1-\alpha^{\prime 2} r^{2}\right) e^{-(1 / 2) \alpha^{\prime 2} r^{2}} .
\end{aligned}
$$

Then, the two steady states we prepared are

$$
\begin{aligned}
& \phi_{0,0}=N_{0,0}\left(\begin{array}{c}
0 \\
0 \\
0 \\
F_{0,0} \\
0 \\
0 \\
\frac{E_{0,0}}{m_{0}} F_{0,0} \\
\frac{\alpha^{\prime} \kappa}{m_{0}} F_{1,1} \\
-\frac{i \alpha^{\prime} \kappa}{m_{0}} F_{1,1} \\
0
\end{array}\right) \text {, } \\
& \phi_{2,0}=N_{2,0}\left(\begin{array}{c}
0 \\
0 \\
0 \\
F_{2,0} \\
0 \\
0 \\
\frac{E_{2,0}}{m_{0}} F_{2,0} \\
\frac{\alpha^{\prime} \kappa}{m_{0}}\left(\sqrt{2} F_{3,1}+F_{1,-1}\right) \\
-\frac{i \alpha^{\prime} \kappa}{m_{0}}\left(\sqrt{2} F_{3,1}-F_{1,-1}\right) \\
0
\end{array}\right) .
\end{aligned}
$$

Superposing these two steady states homogeneously, we get the superposition state which takes the same form as (15). Then the minimum time for this state evolving from the initial state $\Psi(r, \varphi, 0)$ to the orthogonal final one $\Psi\left(r, \varphi, T_{\min }\right)$ is identical to (45). The displacement along the radial direction can also be calculated directly. It is equivalent to (46) in the limit of $B \rightarrow \infty$. Thus, the average speed of the wave packet during interval $\left[0, T_{\min }\right]$ is given by (47). Compared with the commutative counterpart, there is an extra factor $|\kappa|=|1-q \theta B / 4|$ which enables the average speed along the radial direction to exceed light in vacuum. Therefore, it means 
that Lorentz invariance is also violated in this relativistic spin1 boson due to noncommutativity.

\section{Conclusions and Remarks}

In this paper, we investigate the problem of whether Lorentz invariance is violated in noncommutative relativistic quantum mechanics regime. It is known that there are two frames in studying the noncommutative theories $[55,56]$. One is the $\mathrm{C}$-frame, in which one works in the commutative space by mapping the noncommutative variables into commutative ones. The other frame is the NC-frame, in which one works in the noncommutative phase spaces (31) and (32) directly. Since we have to calculate the wave functions explicitly, it is more convenient to work in the $\mathrm{C}$-frame in our studies. It is an interesting task to study this problem in NC-frame directly.

In fact, the violation of Lorentz invariance due to noncommutativity has been noticed for more than 10 years in [45-47]. Nevertheless, there is a difference between [46, 47] and ours. In $[46,47]$, the authors investigate the propagation of electromagnetic wave in noncommutative space. The electromagnetic wave, from the quantum point of view, is photons, which are massless.

We study the charged massive spin- 0 and spin- 1 relativistic bosons in the presence of homogeneous magnetic fields in 2-dimensional space. Both commutative and noncommutative cases are studied. According to the theory of special relativity, the speed of a massive particle can not exceed the speed of light in vacuum. In our studies, we find that the average radial speeds of wave packets during the interval $\left[0, T_{\min }\right]$ in commutative plane are less than the speed of light in vacuum, no matter how strong the intensity of the magnetic field is. However, when noncommutativity is taken into account, we find that the average radial speed takes the form $\bar{v} \doteq 0.5254|\kappa|=0.5254|1-q \theta B / 4|$, which will exceed the speed of light in vacuum if the intensity of the magnetic field is strong enough. It conflicts with the special relativity directly. Therefore, it indicates that Lorentz invariance will be violated in noncommutative space in quantum mechanics regime.

It is known that there are redundant degrees of freedom in DKP equation. For the spin-0 case, the physical degree of freedom is easy to obtain. However, it is not straightforward to get the degrees of freedom for spin-1 case [57]. The solutions (28) and (51) are based on $\psi^{2}=\psi^{3}=0$, which are special solutions to DKP equation. It may be worthwhile to study whether the same conclusions will still be held for the general solutions of the DKP equation.

\section{Conflicts of Interest}

The authors declare that there are no conflicts of interest regarding the publication of this paper.

\section{Acknowledgments}

This work is supported by the NSFC with Grant nos. 11775303 and 11465006.

\section{References}

[1] G. Petiau, “Acad. Roy. Belg," A. Sci., Mem. Collect, vol. 16, 1936.

[2] R. J. Duffin, "On the characteristic matrices of covariant systems," Physical Review, vol. 54, no. 12, p. 1114, 1938.

[3] N. Kemmer, Proc. R. Soc. A, vol. 173, 1939.

[4] S. Zarrinkamar, A. A. Rajabi, H. Rahimov, and H. Hassanabadi, Mod. Phys. Lett. A, vol. 26, p. 1621, 2011.

[5] B. Boutabia-Cheraitia and T. Boudjedaa, "Solution of DKP equation in Woods-Saxon potential," Physics Letters. A, vol. 338, no. 2, pp. 97-107, 2005.

[6] K. Sogut and A. Havare, Phys. Scr, vol. 8, p. 45013, 2010.

[7] Y. Chargui, A. Trabelsi, and L. Chetouani, "Bound-states of the $(1+1)$-dimensional DKP equation with a pseudoscalar linear plus Coulomb-like potential," Physics Letters. A, vol. 374, no. 29, pp. 2907-2913, 2010.

[8] L. Chetouani, M. Merad, T. Boudjedaa, and A. Lecheheb, "Solution of Duffin-Kemmer-Petiau equation for the step potential," International Journal of Theoretical Physics, vol. 43, no. 4, pp. 1147-1159, 2004.

[9] M. Nowakowski, "The electromagnetic coupling in KemmerDuffin-Petiau theory," Physics Letters. A, vol. 244, no. 5, pp. 329337, 1998.

[10] L. Mandelstam and I. G. Tamm, J. Phys, URSS, vol. 9, 1945.

[11] G. N. Fleming and N. A. Cimento, "Phys. Rev. Lett," Am. J. Phys, vol. 6, 1973.

[12] N. Margolus and L. B. Levitin, "The maximum speed of dynamical evolution," Physica D: Nonlinear Phenomena, vol. 120, no. 1-2, pp. 188-195, 1998.

[13] V. Giovannetti, S. Lloyd, and L. Maccone, "Quantum private queries: security analysis," Institute of Electrical and Electronics Engineers. Transactions on Information Theory, vol. 56, no. 7, pp. 3465-3477, 2010.

[14] L. B. Levitin and T. Toffoli, "Fundamental Limit on the Rate of Quantum Dynamics: The Unified Bound Is Tight," Physical Review Letters, vol. 103, no. 16, 2009.

[15] D. V. Villamizar and E. I. Duzzioni, "Quantum speed limit for a relativistic electron in a uniform magnetic field," Physical Review A, vol. 92, no. 4, 2015.

[16] J. Polchinski, Superstring Theory and Beyond String Theory, vol. 2 of Cambridge, UK, Cambridge University Press, 1998.

[17] A. Bilal and C.-S. Chu, "Nucl. Phys," Nuclear Physics. B. Theoretical, Phenomenological, and Experimental High Energy Physics. Quantum Field Theory and Statistical Systems, vol. 547, no. 1-2, pp. 179-200, 1999.

[18] F. Ardalan, H. Arfaei, and M. M. Sheikh-Jabbari, "Dirac quantization of open strings and noncommutativity in branes," Nuclear Physics. B. Theoretical, Phenomenological, and Experimental High Energy Physics. Quantum Field Theory and Statistical Systems, vol. 576, no. 1-3, pp. 578-596, 2000.

[19] J. Jing and Z.-W. Long, "Open string in the constant $B$-field background," Physical Review. D. Third Series, vol. 72, no. 12, 126002, 6 pages, 2005.

[20] A. Connes, M. Douglas, and A. S. Schwarz, "KATP channel formation by the sulphonylurea receptors SUR1 with Kir6.2 subunits in rat dorsal vagal neurons in situ," The Journal of Physiology, vol. 509, p. 2, 1998.

[21] N. Seiberg, E. Witten, and J. High Energy, Phys, vol. 9, 1999.

[22] M. R. Douglas and N. A. Nekrasov, "Noncommutative field theory," Reviews of Modern Physics, vol. 73, no. 4, pp. 977-1029, 2001. 
[23] S. Minwalla, M. Van Raamsdond, N. Seiberg, and J., High Energy Phys, vol. 2, p. 020, 2000.

[24] M. Van Raamsdond and N. Seiberg, J. High Energy Phys, vol. 3, p. 35, 2000.

[25] R. Gopakumar, S. Minwalla, and A. Strominger, "Noncommutative solitons," https://arxiv.org/abs/hep-th/0003160.

[26] M. Chaichian, M. M. Sheikh-Jabbari, and A. Tureanu, "Hydrogen atom spectrum and the lamb shift in noncommutative QED," Physical Review Letters, vol. 86, no. 13, pp. 2716-2719, 2001.

[27] M. Chaichian, P. Presnajder, M. M. Sheikh-Jabbari, and A. Tureanu, "Non-commutative standard model: model building," The European Physical Journal C. Particles and Fields, vol. 29, no. 3, pp. 413-432, 2003.

[28] M. Chaichian, A. Demichev, P. Prešnajder, M. M. SheikhJabbari, and A. Tureanu, "Quantum theories on noncommutative spaces with nontrivial topology: Aharonov-Bohm and Casimir effects," Nuclear Physics B, vol. 611, no. 1-3, pp. 383-402, 2001.

[29] B. Morariu and A. P. Polychronakos, "Quantum mechanics on the noncommutative torus," Nuclear Physics. B. Theoretical, Phenomenological, and Experimental High Energy Physics. Quantum Field Theory and Statistical Systems, vol. 610, no. 3, pp. 531-544, 2001.

[30] D. Karabali, V. P. Nair, and A. P. Polychronakos, "Spectrum of Schrodinger field in a noncommutative magnetic monopole," Nuclear Physics. B. Theoretical, Phenomenological, and Experimental High Energy Physics. Quantum Field Theory and Statistical Systems, vol. 627, no. 3, pp. 565-579, 2002.

[31] B. Morariu and A. P. Polychronakos, "Quantum mechanics on noncommutative Riemann surfaces," Nuclear Physics. B. Theoretical, Phenomenological, and Experimental High Energy Physics. Quantum Field Theory and Statistical Systems, vol. 634, no. 1-2, pp. 326-338, 2002.

[32] C. Acatrinei, "Path integral formulation of noncommutative quantum mechanics," Journal of High Energy Physics, vol. 2001, no. 09, pp. 007-007, 2001.

[33] T. C. Adorno, M. C. Baldiotti, M. Chaichian, D. M. Gitman, and A. Tureanu, "Dirac equation in noncommutative space for hydrogen atom," Physics Letters. B. Particle Physics, Nuclear Physics and Cosmology, vol. 682, no. 2, pp. 235-239, 2009.

[34] K. Ma, J. H Wang, H. X Yang, and B. Phys. Lett, Phys. Lett. B, p. 221, 2016.

[35] K. Ma, J. H Wang, and H. X Yang, Phys. Lett. B, vol. 759, p. 306, 2016.

[36] K. Ma, J. H. Wang, and H. X. Yang, "Probing the noncommutative effects of phase space in the time-dependent AharonovBohm effect," Annals of Physics, vol. 383, pp. 120-129, 2016.

[37] Z. Wang, Z. W. Long, C. Y. Long, and W. Zhang, Adv. High Energy Phys, vol. 201, Article ID 901675, 2015.

[38] B. Q. Wang, Z. W. Long, C. Y. Long, and S. R. Wu, Adv. High Energy Phys, vol. 201, Article ID 2843020, 2017.

[39] G. Guo, C. Long, Z. Yang, and S. Qin, "DKP oscillator in noncommutative phase space," Canadian Journal of Physics, vol. 87, no. 9, pp. 989-993, 2009.

[40] Z.-H. Yang, C.-Y. Long, S.-J. Qin, and Z.-W. Long, "DKP oscillator with spin-0 in three-dimensional noncommutative phase space," International Journal of Theoretical Physics, vol. 49, no. 3, pp. 644-651, 2010.

[41] M. Falek and M. Merad, "A generalized bosonic oscillator in the presence of a minimal length," Journal of Mathematical Physics, vol. 51, no. 3, 033516, 15 pages, 2010.
[42] G. R. de Melo, M. de Montigny, and E. S. Santos, Int. J. Theor. Phys, vol. 2012

[43] M. Chaichian, D. Ellinas, and P. Kulish, "Quantum algebra as the dynamical symmetry of the deformed Jaynes-Cummings model," Physical Review Letters, vol. 65, no. 8, pp. 980-983, 1990.

[44] K. Wang, Y. F. Zhang, S. J. Liu, Z. W. Long, and J. Jing, "Quantum speed limit for a relativistic electron in the noncommutative phase space," High Energy Physics - Theory, vol. 32, no. 23n24, 2017.

[45] S. M. Carroll, J. A. Harvey, V. . Kostelecky, C. D. Lane, and T. Okamoto, "Noncommutative field theory and Lorentz violation," Physical Review Letters, vol. 87, no. 14, Article ID 141601, 141601, 4 pages, 2001.

[46] Z. Guralnik, R. Jackiw, S. Y. Pi, and A. P. Polychronakos, "Testing non-commutative QED, constructing non-commutative MHD," Physics Letters, Section B: Nuclear, Elementary Particle and High-Energy Physics, vol. 517, no. 3-4, pp. 450-456, 2001.

[47] R. G. Cai, Phys. Lett. B, vol. 517, p. 457, 2001.

[48] A. A. Deriglazov and W. G. Ramirez, "World-line geometry probed by fast spinning particle," Modern Physics Letters A. Particles and Fields, Gravitation, Cosmology, Nuclear Physics, vol. 30, no. 21, Article ID 1550101, 2015.

[49] A. A. Deriglazov and W. G. Ramrez, Int. J. Mod. Phys. D, vol. 26, Article ID 1750047, 2017.

[50] A. A. Deriglazov and W. G. Ramrez, Adv. HEP, vol. 2016, Article ID 1376016, 2016.

[51] D. Yoshioka, The quantum Hall effect, vol. 133 of Springer Series in Solid-State Sciences, Springer, Berlin, Germany, 2002.

[52] J. Gamboa, M. Loewe, F. Mendez, and J. C. Rojas, "Noncommutative quantum mechanics," Physical Review D, vol. 64, no. 6 , Article ID 067901, 2001.

[53] O. Bertolami, J. G. Rosa, C. M. L. de Aragao, P. Castorina, and D. Zappala, "Berry phase in the gravitational quantum well and the Seiberg-Witten map," Physics Letters. A, vol. 372, no. 34, pp. 5556-5559, 2008.

[54] J. Zhang, K. Gao, and C. Ning, "Test of quantum effects of spatial noncommutativity using modified electron momentum spectroscopy," Physical Review D, vol. 78, no. 10, 2008.

[55] G. D. Barbosa, "Noncommutative conformally coupled scalar field cosmology and its commutative counterpart," Physical Review. D. Third Series, vol. 71, no. 6, 063511, 14 pages, 2005.

[56] S. Perez-Payan, M. Sabido, E. Mena, and C. Yee-Romero, "Analysis of scalar field cosmology with phase space deformations," Advances in High Energy Physics, vol. 2014, Article ID 958137, 2014.

[57] T. R. Cardoso, L. B. Castro, and A. S. de Castro, "Inconsistencies of a purported probability current in the Duffin-KemmerPetiau theory," Physics Letters. A, vol. 372, no. 38, pp. 5964-5967, 2008 . 

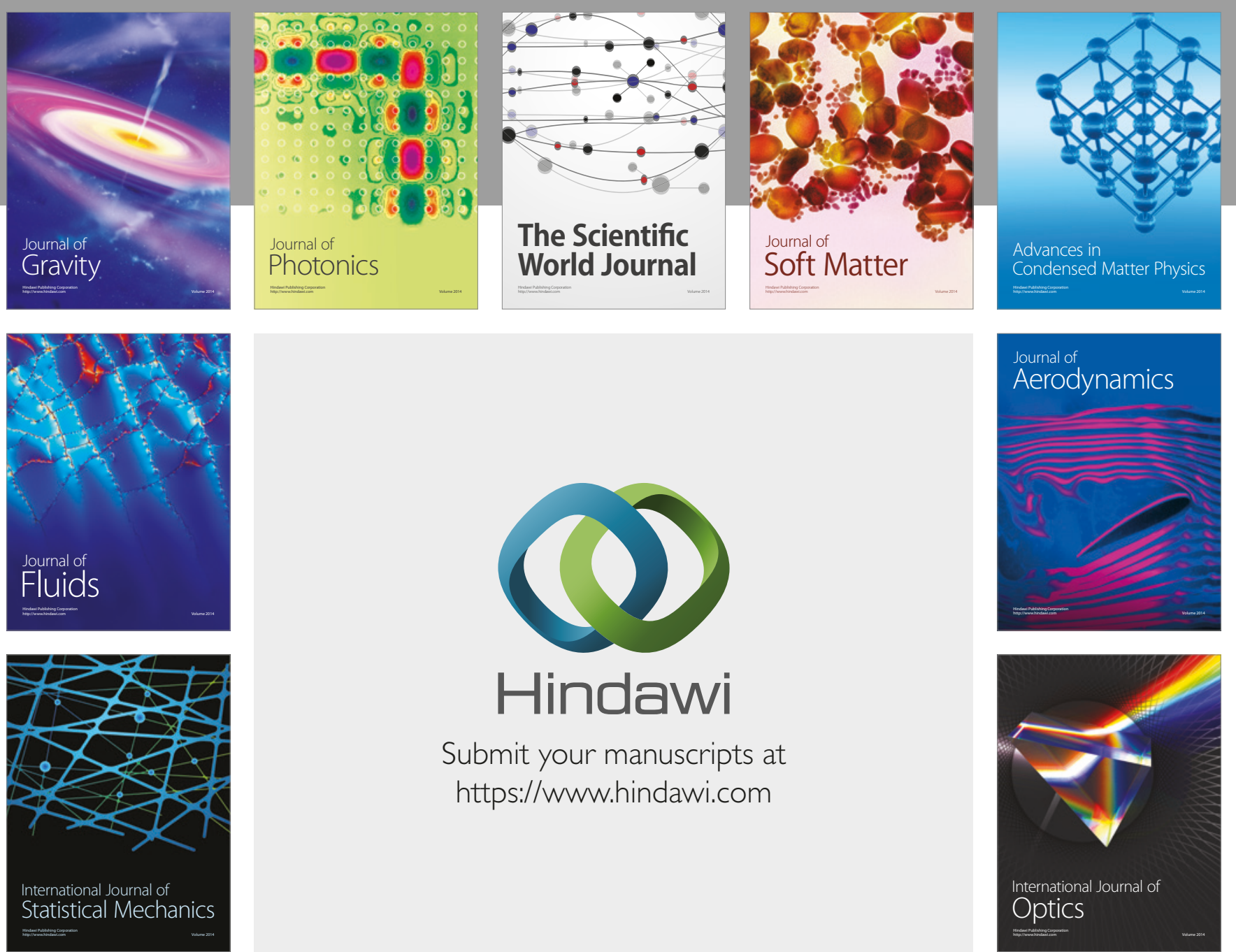

Submit your manuscripts at

https://www.hindawi.com
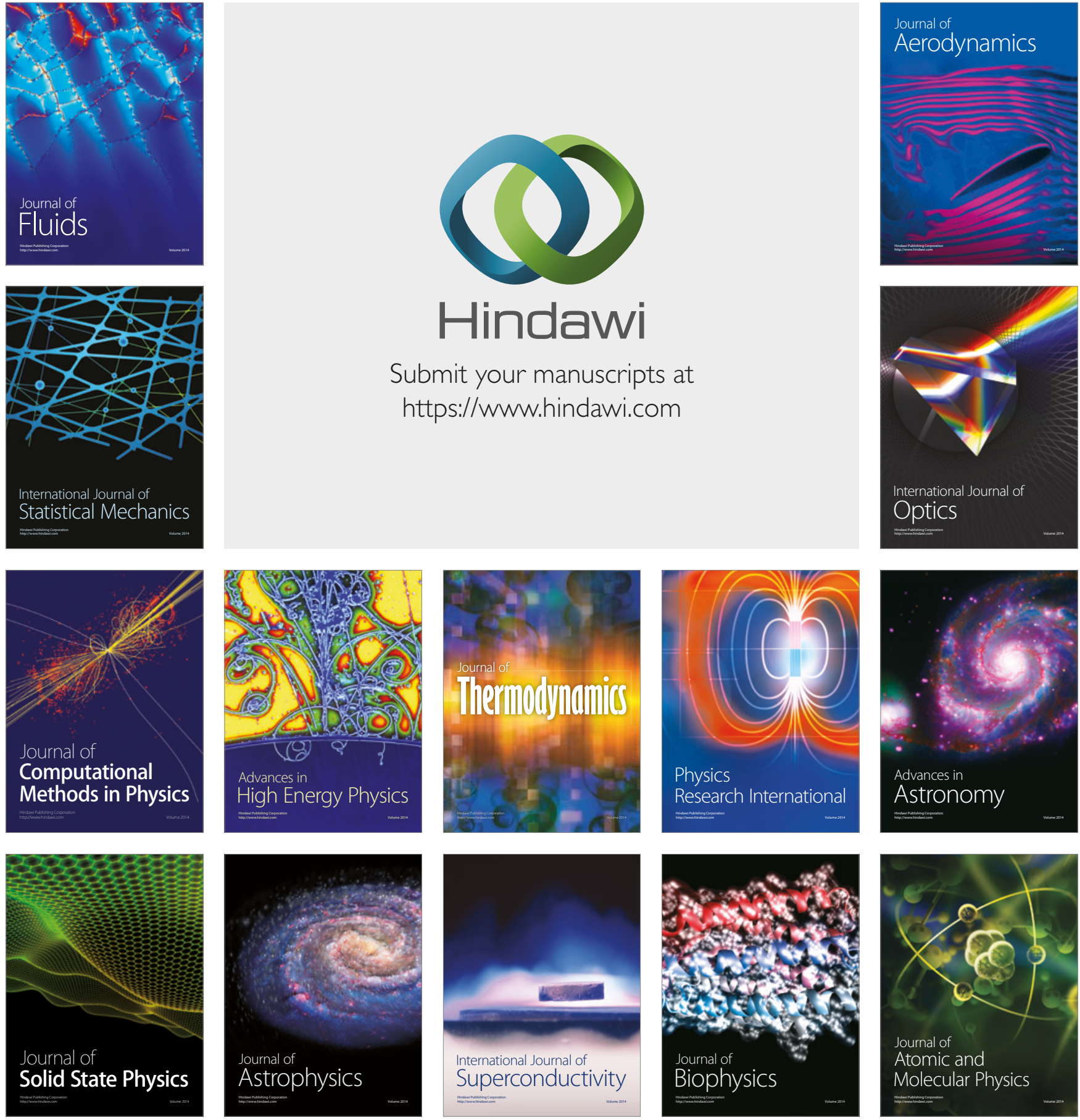